Review Article

\title{
Natural Products in Therapeutic Management of Multineurodegenerative Disorders by Targeting Autophagy
}

\author{
Sibhghatulla Shaikh, ${ }^{1,2}$ Khurshid Ahmad ${ }^{1,2}$ Syed Sayeed Ahmad, ${ }^{1,2}$ Eun Ju Lee,, \\ Jeong Ho Lim, ${ }^{1}$ Mirza Masroor Ali Beg $\left(\mathbb{1}^{3},{ }^{3}\right.$ Amit K. Verma, ${ }^{4}$ and Inho Choi $i^{1,2}$ \\ ${ }^{1}$ Department of Medical Biotechnology, Yeungnam University, Gyeongsan 38541, Republic of Korea \\ ${ }^{2}$ Research Institute of Cell Culture, Yeungnam University, Gyeongsan 38541, Republic of Korea \\ ${ }^{3}$ Faculty of Medicine, Ala-Too International University, Bishkek, Kyrgyzstan \\ ${ }^{4}$ Department of Biotechnology, Jamia Millia Islamia, New Delhi, India
}

Correspondence should be addressed to Inho Choi; inhochoi@ynu.ac.kr

Sibhghatulla Shaikh and Khurshid Ahmad contributed equally to this work.

Received 7 May 2021; Revised 9 August 2021; Accepted 18 August 2021; Published 14 September 2021

Academic Editor: Xinfeng Li

Copyright (c) 2021 Sibhghatulla Shaikh et al. This is an open access article distributed under the Creative Commons Attribution License, which permits unrestricted use, distribution, and reproduction in any medium, provided the original work is properly cited.

\begin{abstract}
Autophagy is an essential cellular process that involves the transport of cytoplasmic content in double-membraned vesicles to lysosomes for degradation. Neurons do not undergo cytokinesis, and thus, the cell division process cannot reduce levels of unnecessary proteins. The primary cause of neurodegenerative disorders (NDs) is the abnormal deposition of proteins inside neuronal cells, and this could be averted by autophagic degradation. Thus, autophagy is an important consideration when considering means of developing treatments for NDs. Various pharmacological studies have reported that the active components in herbal medicines exhibit therapeutic benefits in NDs, for example, by inhibiting cholinesterase activity and modulating amyloid beta levels, and $\alpha$-synuclein metabolism. A variety of bioactive constituents from medicinal plants are viewed as promising autophagy controllers and are revealed to recover the NDs by targeting the autophagic pathway. In the present review, we discuss the role of autophagy in the therapeutic management of several NDs. The molecular process responsible for autophagy and its importance in various NDs and the beneficial effects of medicinal plants in NDs by targeting autophagy are also discussed.
\end{abstract}

\section{Introduction}

Autophagy is a fundamental, exceptionally well-controlled process in the eukaryotic cell recycling system under different states of cellular stress. Autophagy plays an essential role in cell survival and maintenance by degrading cytoplasmic organelles, macromolecules, and misfolded proteins and, thus, facilitates the recycling of cellular content as breakdown products $[1,2]$. The literal meaning of autophagy is "self-eating," and this process involves the intracellular capture of unnecessary proteins, lipids, and organelles and directing them for their further degradation in the lysosomal compartment [3]. Thus, autophagy performs a vital role in the overall homeostasis of proteins and, eventually, cells and retains metabolic balance between the synthesis and degradation of cytoplasmic materials and their subsequent reprocessing. Thus, autophagy is a natural cellular mechanism that sustains cellular homeostasis under various conditions [4]. Many other functions of autophagy have been identified in the presence of pathological processes such as infectious diseases, myocardial diseases, diabetes, neurodegenerative diseases (NDs), and various cancers $[5,6]$.

There are typically three types of autophagy: macroautophagy, microautophagy, and chaperone-mediated autophagy (CMA), all of which have specific roles that depend on the cellular microenvironment, signals, and organs, but all usually involve proteolytic degradation of cytosolic components inside lysosomes [7]. Macroautophagy is the most 
important degradation pathway and involves the formation of double-membrane vesicles called autophagosomes in cytoplasm. In fact, the term "autophagy" typically refers to this process, unless otherwise specified. Macroautophagy may further be classified as mitophagy, nucleophagy, pexophagy, aggrephagy, and xenophagy [8]. Pathological studies have shown that macroautophagy deficiencies may be involved in the development of NDs [9]. In microautophagy, cellular constituents are taken up by lysosomes by direct engulfment, projection, or septation of lysosome membranes [10]. Macro- and microautophagy are both capable of engulfing large cellular components through selective and nonselective mechanisms. CMA is an extensive form of autophagy found in almost all cells and tissue types in higher eukaryotes and involves the direct recognition of targeted proteins containing the KFERQ motif, which is recognized by chaperone proteins, such as the heat shock protein (Hsc-70). These proteins are then delivered directly to lysosomes via the lysosomal-associated membrane protein $2 \mathrm{~A}$ (LAMP-2A) [11, 12]. Studies increasingly support the notion that dysregulation of the CMA pathway plays a role in multiple NDs including Alzheimer's Disease (AD), Parkinson's Disease (PD), Amyotrophic Lateral Sclerosis (ALS), and Frontotemporal Lobar Degeneration [12].

Neurons are of the postmitotic cell type and do not go through cytokinesis, and thus, cell division cannot reduce unnecessary deposited proteins. Therefore, the primary cause of NDs is pathological protein accumulation within neuronal cells, and this could be prevented by autophagic degradation. Hence, autophagy activation plays an important role in the treatment of NDs and provides a strategic platform for disease management $[13,14]$. In this review, we discussed the beneficial effects of medicinal plants on NDs by targeting autophagy. Before describing the roles of herbal products in NDs, we provide a brief overview of autophagic processes at a molecular level and their roles in various NDs.

\section{Molecular Process of Autophagy}

Autophagy is highly inducible by starvation and other stressrelated responses, and its activation causes a rapid increase in numbers of autophagosomes, which are generated on or near the endoplasmic reticulum [15]. In addition, membranes acquired from the Golgi complex, mitochondria, and plasma membrane also contribute to autophagosome formation [15]. Autophagosome establishment involves a multicomponent complex process, which is governed by multiple Atg proteins and related proteins. Of the 35 Atg proteins known, Atg1-10, 12-14, 16, and 18 are the "core Atg proteins" in yeast [16], and these proteins with Atg17, 29, and 31 participate in autophagosome formation. Other autophagic pathways such as pexophagy (autophagic peroxisome degradation) and cytoplasm-to-vacuole targeting share core Atg proteins $[17,18]$. Furthermore, core Atg proteins are highly conserved in eukaryotes, including mammals, and function in a similar hierarchical manner in yeast [19].
Studies have shown that mTOR is essential for promoting adult stem cell differentiation, progenitor cell growth, and proliferation and has a great impact on the multipotent stem cell population $[20,21]$. Interruption or deregulation of the autophagy system has been implicated in neurodegenerative issues such as $\mathrm{AD}$, and $\mathrm{A} \beta$ assumes a significant role in this autophagy framework. Autophagy plays a substantial role in the generation and digestion of $\mathrm{A} \beta$ and the accumulation of tau. Much of the time, autophagy is directed by the phosphatidylinositol 3-phosphate kinase/AKT/mTOR/p70 ribosomal protein S6 kinase signaling pathway [22].

Various mTOR-dependent and mTOR-independent autophagy modulators have been found to have beneficial effects in the management of AD [23]. The mTOR pathway appears to be an adaptable player, and its modulation can affect both neuroprotective and cognitive processes. Although direct targeting of mTOR does not appear to have therapeutic potential, its indirect modulation by other signaling pathways is encouraging in this respect [24]. Targeting of rapamycin complex 1 (mTORC1) promotes cell growth in nutrient-rich environments by inducing the biosynthesis of proteins, lipid, and nucleotides and inhibiting cellular catabolism by suppressing the autophagic pathway $[25,26]$. Aberrant mTOR signaling is associated with brain abnormalities and NDs. Even subtle defects in the mTOR pathway may have severe effects, including neurological and psychiatric disorders. Conversely, mTOR inhibitors can be useful in several neuropsychiatric alterations such as in brain cancer, brain ischemia, schizophrenia, autism, and NDs. mTOR has been linked to synaptic plasticity and the activation of autophagy [27]. Under nutrient-rich conditions, by direct association with the ULK1-Atg13-FIP200 complex, mTOR suppresses autophagy and mediates the phosphorylation-dependent inhibitions of the autophagy-related kinase activities of -13 (Atg13) and Unc-51-like kinase 1 (ULK1). Furthermore, the mTOR-mediated phosphorylations of Atg13 and ULK1 are inhibited under starvation conditions or after treatment with rapamycin. Rapamycin inhibits $\mathrm{mTOR}$, and this triggers autophagy by dephosphorylating ULK1, ULK2, and Atg13 and activating ULK to phosphorylate FIP200 (FAK familyinteracting protein of $200 \mathrm{kDa})[28,29]$. The existence of mTOR-independent regulation of mammalian autophagy resulted from the observation that intracellular inositol and inositol 1,4,5-trisphosphate (IP3) levels negatively regulate autophagy [30]. Furthermore, inhibition of inositol monophosphatase decreased levels of free inositol and IP3, which led to autophagy upregulation [31].

In postmitotic neuronal cells, basal autophagy movement is important, possibly because of their inability to dilute noxious components via cell division [32]. Autophagic activity is enhanced by various burdens, for example, by supplement starvation, hypoxia, or inflammation [33]. During certain physiological processes and pathological conditions, increased autophagy leads to cell death, the elimination of microorganisms entering cells, and cancer suppression [34]. Then again, the reduced autophagic potential is related to aging [35]. During autophagy, proteins are degraded into amino acids and, thus, provide a source of energy and raw materials for protein synthesis [36]. Hence, the dysregulation of autophagy may 
result in the aggregation of intracellular proteins. Furthermore, different types of autophagy dysfunctions can lead to $\mathrm{ND}$ or ND-like symptoms, such as the inhibition of autophagosome-lysosome fusion [37], reduced lysosomal acidification [38], or intracellular protein deposition [39].

Two complexes, that is, mTORC1 and mTORC2, are responsible for the regulation of autophagy. mTORC1 consists of four different protein factors, viz., raptor (regulatory-associated protein of mTOR), deptor (DEP-domain containing mTOR-interacting protein), PRAS40 (proline-rich Akt substrate of $40 \mathrm{kDa}$ ), and mLST8 (mammalian lethal with SEC13 protein 8), whereas mTORC2 consists of rictor (rapamycin insensitive companion of mTOR), protor (protein observed with rictor), and mSIN1 (mammalian stressactivated mitogen-activated protein kinase-interacting protein) along with mLST8 and deptor $[40,41]$. Starvation results in the activation of the mTORC1 complex, which stimulates autophagy resulting in the recycling of intracellular components as a source of energy [42]. In addition, the phosphorylation of Akt by the mTORC2 complex results in the activation of the mTORC1 complex (Figure 1).

\section{Autophagy, Immune Response, and Neurodegeneration}

Most cellular stress-response pathways, including those that regulate immunological responses and inflammation, interact with the autophagy machinery [43-45]. The autophagy pathway/proteins have a complex reciprocal relationship with immunity and inflammation; autophagy proteins are involved in both the stimulation and suppression of immune and inflammatory responses, and immune and inflammatory signals are involved in both the stimulation and suppression of autophagy [44].

Autophagic interference with type I interferon responses occurs either directly by targeting signaling molecules within the pathway, beginning with RIG-I-like receptors or cGAMP synthase (a cytosolic DNA sensor) and progressing to the stimulator of the interferon gene (STING) and interferon regulatory factors, or indirectly by removing agonist sources that activate these pathways [46-49]. The p62 receptor appears to have a function in preventing $\mathrm{T}$-cell receptor(TCR-) mediated NF- $\kappa \mathrm{B}$ signaling via Bcl10. Although p62 initiates signaling, it also functions as a receptor to degrade Bcl10, which becomes ubiquitinated in response to TCR activation. Therefore, this strategy may protect cells against NF- $\kappa$ B hyperactivation as a result of TCR signaling [50].

Using fly genetics, researchers show that deregulation of cyclin-dependent kinase 5 (Cdk5) activity disrupts autophagy, leads to an overactive innate immune response, and results in dopamine neurodegeneration in Drosophila [51]. It was demonstrated that an overactive innate immune response was sufficient to trigger neuronal cell death. Intriguingly, inhibiting the $\mathrm{NF}-\kappa \mathrm{B}$ transcription factor in neurons lowers neuronal loss and downregulates the innate immune response genes in the Cdk5-deficient background [52].

Many NDs are linked with inflammatory responses in glia, which may contribute to pathology, and autophagy in glial cells may play a role in regulating these processes [53]. Microglia, as a key immune cell in the brain, influences phagocytosis and inflammation in age-related NDs [54]. When the LC3B and Atg7 genes were inhibited, microglia failed to degrade extracellular $\mathrm{A} \beta$, indicating that autophagy function impairment in microglia may contribute to CNSdegenerative neurological disease [55]. Astrocytes are specialized glial cells in the brain and spinal cord and have been linked to the development of various NDs such as $\mathrm{AD}, \mathrm{PD}$, and ALS [56, 57]. Trifluoperazine-induced autophagy was implicated in astrocyte protection against bilirubin-induced cytotoxicity [58]. Recently, it has been demonstrated that Atg5 knockdown reduced astrocyte development in vivo, but Atg5 overexpression resulted in excessive astrocyte differentiation in vivo [59].

\section{Autophagy and Neurodegenerative Disorders}

4.1. Autophagy in Alzheimer's Disease (AD). AD is characterized by the depositions of $\mathrm{A} \beta$ and tau in the brain. Under normal conditions, the production and clearance rates of $\mathrm{A} \beta$ are balanced, and $\mathrm{A} \beta$ is not deposited inside neuronal cells. Enhanced aggregation of $\mathrm{A} \beta$ peptides has been found in $\mathrm{AD}$ patients, and it is well recognized that failure of the autophagic system is a characteristic of AD. Recently, it was shown that autophagy enhanced the protein degradations of $\mathrm{A} \beta$ and tau [60]. During autophagy, autophagosomes enclosing $\mathrm{A} \beta$ facilitate its degradation by fusing with lysosomes. In addition, the microglial inflammatory response is regulated by autophagy, and dysregulation of autophagy damages neurons by exacerbating NLRP3 inflammasome signaling [55].

The C-terminal fragments of the amyloid precursor protein (APP) might be an etiological trigger for $\mathrm{AD}$ [61]. The cleavage of APP by BACE-1 produces C99 fragments. Reductions in autophagy (inhibition of autophagosome production or prevention of autophagosome fusion with lysosomes) result in increased C99 levels [62, 63]. Conversely, enhanced autophagy, either by mTOR suppression or by starvation, enhances C99 clearance in degenerative lysosomes $[62,64]$. Also, lysosomes are disrupted by phagocytosis of the $\mathrm{A} \beta$ peptide, which results in the release of a lysosomal proteolytic enzyme (cathepsin B), which, in turn, stimulates pyrin domain containing 3 inflammasomes and leads to the production of proinflammatory and neurotoxic factors via the interleukin 1 beta pathway [65]. Stimulation of the autophagic system via cystatin B deletion decreases $\mathrm{A} \beta$ aggregation and has protective effects in mouse models of $\mathrm{AD}$ [66]. Tau protein stabilizes the microtubule, but its hyperphosphorylation reduces its affinity for microtubules and results in microtubule entanglement. Thus, the elimination of the tau protein by the autophagic system is required to address NDs $[67,68]$. In addition, enhanced accumulation of the tau protein in the presence of the autophagic inhibitor (3-methyladenine) suggests that autophagy is required to prevent tau aggregation [69].

Various approaches used to upregulate the autophagic system have potential use for the management of $\mathrm{AD}$ [70]. Rapamycin, an inhibitor of the mTOR pathway, decreased 


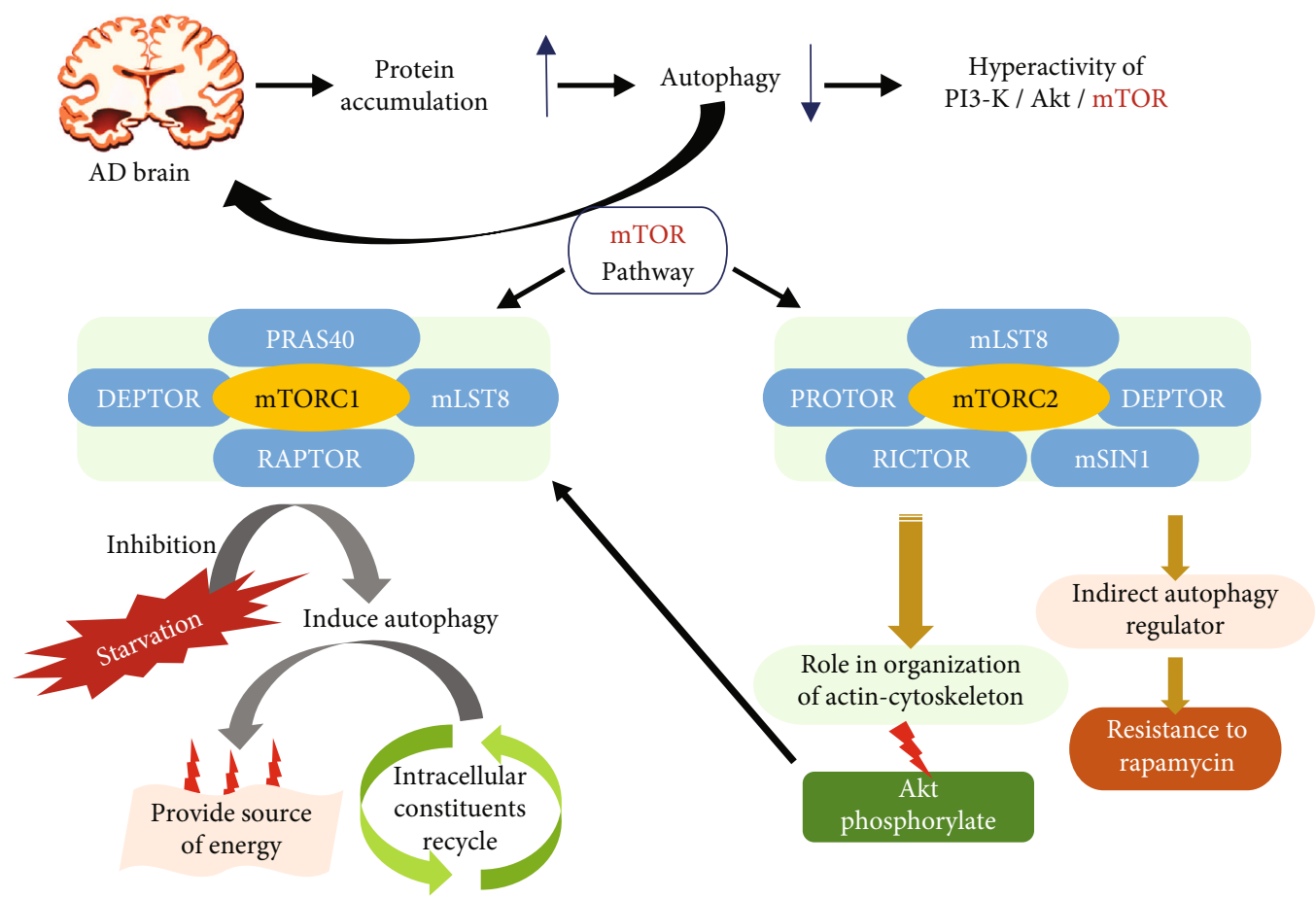

Figure 1: Molecular pathways in autophagy.

$\mathrm{A} \beta$ deposition and prevented $\mathrm{AD}$ development by enhancing autophagy in an animal model of $\mathrm{AD}[71,72]$, and the multifunctional protein p62 has been linked to neuropathological inclusions in various NDs and with the degradations of $\mathrm{A} \beta$ and tau. The ubiquitin-binding domain and the LC3(microtubule-associated protein 1 light chain 3-) interacting regions of p62 are two functional domains. Enhancing brain p62 expression promoted autophagy and led to cognitive improvement in an animal model of $\mathrm{AD}$, whereas removing the LC3-interacting region domain disrupted $\mathrm{A} \beta$ clearance by preventing autophagy [73]. In another study, latrepirdine was found to decrease $\mathrm{A} \beta$ aggregation by stimulating the Atg5-dependent autophagy in an animal model [70]. These reports indicate that autophagy is disrupted in $\mathrm{AD}$ and that regulating the autophagic system offers a reasonable therapeutic approach.

4.2. Autophagy in Parkinson's Disease (PD). Autophagy and the ubiquitin-mediated pathway eliminate misfolded proteins in healthy cells, but both of these pathways are disrupted in $\mathrm{PD}$, which results in the aggregation of misfolded proteins [74]. One of the important hallmarks of PD is the deposition of misfolded $\alpha$-synuclein into intraneuronal inclusions known as Lewy bodies (LBs). $\alpha$-Synuclein is susceptible to degradation by chaperone-mediated autophagy [75]. In familial PD, lysosomes are unable to engulf the mutant $\alpha$-synuclein because of its high affinity for the lysosomal receptor (LAMP-2A), which in turn prevents $\alpha$-synuclein degradation by shielding the substrate from CMA [76]. Furthermore, autophagosome and dysfunctional lysosome accumulations were found in postmortem PD brain samples [77], which highlighted the pathogenic role of autophagy in PD. Enhanced $\alpha$-synuclein levels have been found in the lysosomal dysfunction, indicating a close link between autophagy and $\alpha$-synuclein degradation. Various studies have reported that autophagy can degrade all forms of $\alpha$ synuclein $[77,78]$ and that proteasomes also degrades monomeric $\alpha$-synuclein [79].

Overexpression of $\alpha$-synuclein is caused by mutations in SNCA, which encodes for $\alpha$-synuclein, and these are sufficient to cause the progression of PD. Excess $\alpha$-synuclein levels damage the autophagy system by hindering small GTPase Rab-1A [80]. Autophagy contributes in various ways to the protection of neural cells, but $\alpha$-synuclein accumulation enhances protein aggregation levels, counters the effective clearance of misfolded protein, and induces neuronal cell apoptosis [80]. Moreover, $\alpha$-synuclein mutation has been suggested to impair CMA [76, 81]. These results indicate that the regulations of more than one type of autophagy by $\alpha$-synuclein mutations have toxic effects on neuronal cells.

4.3. Autophagy in Amyotrophic Lateral Sclerosis (ALS). ALS is a paralytic condition defined as motor neuronal dysfunctions in the brain and spinal cord resulting in muscle atrophy. Mutations in TAR DNA-binding protein and superoxide dismutase 1 (SOD1) are common causes of familial ALS [82], and it has been established that autophagy is linked with ALS. Rat LC3 is vital for autophagy, and the formation of LC3-II from LC3-I has been suggested to provide a simple means of controlling autophagy. LC3-II overexpression has been reported in mutant SOD1G93A transgenic mice. In addition, enhanced autophagosomes were strongly associated with reduced mTOR phosphorylation in various genetic ALS models [83]. A growing number of studies have established that mutations in autophagy-associated proteins are 
well correlated with the pathogenesis of ALS. The endosomal sorting complexes required for transport (ESCRTs) are responsible for sorting transmembrane proteins into the inner vesicles of the multivesicular body (MVB) during endocytosis. Reductions in ESCRT subunits inhibit either autophagosomeMVB fusion or amphisome-lysosome fusion and are considered to be linked with ALS [84]. Ubiquilin-2 (a proteasome shuttle factor) has an important role in the generation of autophagosomes. Mice with mutated UBQLN2 exhibit neuronal loss, cognitive deficits, and short lifespans [85].

\section{Beneficial Effects of Medicinal Plants on NDs by Targeting Autophagy}

Medicinal herbs have become increasingly important in the quest for more effective and adjunctive treatments [86-89]. Various pharmacological studies have reported that the active components of herbal medicines show therapeutic benefits in NDs via different mechanisms such as by increasing neurogenic activity, inhibiting cholinesterase activity, and controlling $\mathrm{A} \beta$, tau, and $\alpha$-synuclein metabolism by targeting autophagy [90-92]. Table 1 provides a summary of classes of natural compounds that reduce neurodegeneration by regulating autophagy (Figure 2).

5.1. Crude Extracts. Radix Polygalae extract was reported to decrease A $\beta$ and mutant A53T $\alpha$-synuclein levels by activating AMPK/mTOR signaling to stimulate autophagy in Chinese hamster ovary cells (transfected with APP and BACE1) and PC-12 cells, respectively $[93,94]$. Withania somnifera extract had a protective effect in ALS by downregulating p62 (a classical autophagy receptor), thereby promoting autophagy in the motor neurons of SOD $1^{\mathrm{G} 93 \mathrm{~A}}$ mice [95]. In another study, Ginkgo biloba extract repressed microglial inflammation and enhanced cognitive functions by regulating the mechanism moderately involved in the activation of autophagy [96].

5.2. Saponins. Ginsenoside-Rg2, a bioactive compound obtained from Panax ginseng induces autophagy in an AMPK/ULK1-dependent manner. Rg2 increased the clearance of aggregated proteins and enhanced cognitive function by inducing autophagy in an AD mouse model [97]. The protopanaxadiol derivative DDPU (1-(3,4-dimethoxyphenethyl)-3-(3-dehydroxyl-20(s)-protopanaxadiol-3 $\beta$-yl)urea) increased $\mathrm{A} \beta$ clearance by inducing autophagy via the $\mathrm{PI}_{3} \mathrm{~K} / \mathrm{AKT} / \mathrm{mTOR}$ signaling pathway by inhibiting $\mathrm{PI}_{3} \mathrm{~K}$ and decreased $\mathrm{A} \beta$ generation by restraining PERK/eIF $2 \alpha$ signaling-mediated BACE1 translation [98]. In addition, Radix Polygalae derived onjisaponin B enhanced mutant $\alpha$-synuclein degradation by autophagy induction by activating the AMPK/mTOR signaling pathway [93].

5.3. Alkaloids. Alkaloids are the important active components in herbal medicines and exert beneficial effects on NDs by inducing autophagy and inhibiting cholinesterase activity [99, 100]. Alkaloids isolated from Dendrobium nobile enhanced autophagic flux via autophagosome generation and stimulated Beclin-1 expression [101], and berberine has been reported to stimulate autophagy by activating
$\mathrm{Bcl} 2 / \mathrm{Beclin}-1$ signaling, thus increasing $\mathrm{A} \beta$ clearance, and to improve cognitive functions in a mouse model of AD [102]. Furthermore, it has been reported that berberine can bypass the blood-brain barrier [103]. TDP-43 (43 kDa nuclear protein TAR DNA-binding protein) is the main component of ubiquitinated inclusions in aggregated proteins in ALS [104, 105]. Berberine has therapeutic potential in ALS as it reverses TDP-43 proteinopathy by disrupting mTOR/p70S6K signaling and stimulating the autophagic degradation pathway [106]. Corynoxine isolated from Uncaria rhynchophylla is an established inducer of autophagy and enhances autophagosome generation and the elimination of $\alpha$-synuclein in PC12 cells [107]. Isorhynchophylline, a main tetracyclic oxindole alkaloid obtained from U. rhynchophylla, has been used to manage NDs in East Asia for centuries. This alkaloid induces the Beclin-1-dependent autophagy-lysosome pathway and enhances the clearance of $\alpha$-synuclein monomers and $\alpha$-synuclein/synphilin-1 aggresomes from neuronal cells [108]. Angelica sinensis-derived n-butylidenephthalide enhanced motor functions in SOD1-ALS mice. The autophagy pathway was involved in the therapeutic mechanism, as n-butylidenephthalide treatment reduced LC3-II expression and increased mTOR levels [109]. In addition, conophylline from Ervatamia microphylla induced autophagy in Huntington disease and PD models [100, 110].

5.4. Flavonoids. Studies have established that flavonoids influence the autophagy system in some disorders $[111,112]$. Silibinin isolated from Silybum marianum reduced neuronal damage via the BDNF/TrkB pathway by decreasing autophagy in the hippocampus [113]. In another study, wogonin enhanced autophagy by inhibiting the Akt/mTOR pathway and increasing $\mathrm{A} \beta$ clearance [114]. Hesperetin recovered $\mathrm{A} \beta$ damage-induced glucose utilization by downregulating $\mathrm{A} \beta$ stimulated autophagy [115], and kaempferol has been reported to enhance autophagy and decrease ROS, apoptosis, and mitochondrial dysfunction in rotenone-exposed $\mathrm{SH}$ SY5Y cells [116].

5.5. Polyphenols. Curcumin inhibits $\mathrm{A} \beta$ aggregation and ameliorates cognitive functions. The mechanisms responsible involve the stimulation of autophagy by downregulating the $\mathrm{PI}_{3} \mathrm{~K} / \mathrm{Akt} / \mathrm{mTOR}$ pathway [117]. In amyloid-treated HT22 cells, curcumin protected hippocampal neurons by inhibiting the abnormal formation of Beclin-1 and autophagosomes [118]. In an in vitro dopaminergic neuron model of $\mathrm{PD}$, curcumin was involved in the modulation of autophagy and the clearing of $\alpha$-synuclein aggregates [119]. Resveratrol is attracting attention because of its curative potential in AD and has been reported to reduce $\mathrm{A} \beta$ generation and restrain the development of AD by inhibiting apoptosis and regulating mitophagy [120]. Curcumin decreased the accumulation of A53T $\alpha$-synuclein protein (related to early-onset PD) by downregulating $\mathrm{mTOR} / \mathrm{p} 70$ ribosomal protein S6 kinase signaling and induced macroautophagy in SH-SY5Y cells [121]. In addition, resveratrol has been reported to protect against neural damage by activating mitophagy [122] and to stimulate autophagy and lysosomal degradation by regulating the $\mathrm{AMPK} / \mathrm{mTOR}$ signaling pathway and reducing 
TABLE 1: Natural compounds that inhibit neurodegeneration via autophagy.

\begin{tabular}{|c|c|c|c|}
\hline Natural sources & Signaling & Effects & References \\
\hline \multicolumn{4}{|l|}{ Crude extracts } \\
\hline Radix Polygalae & $\mathrm{AMPK} / \mathrm{mTOR}$ & $\begin{array}{c}\text { Decrease } \mathrm{A} \beta \text { and mutant } \mathrm{A} 53 \mathrm{~T} \\
\alpha \text {-synuclein levels }\end{array}$ & {$[93,94]$} \\
\hline Withania somnifera & $\begin{array}{c}\text { Downregulate the p62 } \\
\text { (a classical autophagy receptor) }\end{array}$ & Promote autophagy in motor neuron & {$[95]$} \\
\hline \multicolumn{4}{|l|}{ Saponins } \\
\hline Ginsenoside-Rg2 & $\begin{array}{l}\text { AMPK-ULK1-dependent } \\
\text { and MTOR-independent }\end{array}$ & $\begin{array}{l}\text { Aggregated protein clearance and } \\
\text { enhanced cognitive function }\end{array}$ & {$[97]$} \\
\hline DDPU & $\mathrm{PI} 3 \mathrm{~K} / \mathrm{AKT} / \mathrm{mTOR}$ and PERK/eIF2 $\alpha$ & Clearance of $\mathrm{A} \beta$ and decreased $\mathrm{A} \beta$ generation & {$[98]$} \\
\hline Onjisaponin B & AMPK/mTOR & Enhances mutant $\alpha$-synuclein degradation & {$[93]$} \\
\hline \multicolumn{4}{|l|}{ Alkaloids } \\
\hline Berberine & Bcl2/Beclin-1 & Clearance of $\mathrm{A} \beta$ and improves cognitive function & [102] \\
\hline Isorhynchophylline & Beclin-1 & $\begin{array}{c}\text { Clearance of } \alpha \text {-synuclein monomers and } \\
\alpha \text {-synuclein/synphilin-1 aggresomes }\end{array}$ & [108] \\
\hline n-Butylidenephthalide & mTOR & Enhanced motor functions & [109] \\
\hline \multicolumn{4}{|l|}{ Flavonoids } \\
\hline Silibinin & $\mathrm{BDNF} / \operatorname{TrkB}$ & Reduces neuronal damage & [113] \\
\hline Wogonin & Akt/mTOR & Clearance of $\mathrm{A} \beta$ & {$[114]$} \\
\hline Hesperetin & IRS-PI3K-Akt & Recovers $\mathrm{A} \beta$-damage glucose utilization & {$[115]$} \\
\hline \multicolumn{4}{|l|}{ Polyphenols } \\
\hline Curcumin & $\begin{array}{l}\mathrm{PI} 3 \mathrm{~K} / \mathrm{Akt} / \mathrm{mTOR} \text { and } \mathrm{mTOR} / \mathrm{p} 70 \\
\text { ribosomal protein } \mathrm{S} 6 \text { kinase }\end{array}$ & $\begin{array}{c}\text { Inhibits } \mathrm{A} \beta \text { aggregation, improves cognitive } \\
\text { function and decreased } \mathrm{A} 53 \mathrm{~T} \alpha \text {-synuclein } \\
\text { accumulation }\end{array}$ & {$[117,118]$} \\
\hline Resveratrol & AMPK/mTOR & Decreased $\mathrm{A} \beta$ synthesis & {$[123]$} \\
\hline $\begin{array}{l}2,3,5,4^{\prime} \text {-tetrahydroxystilbene- } \\
\text { 2-O-glycoside }\end{array}$ & Beclin-1 & Cognitive function & [124] \\
\hline Carnosic acid & AMPK/mTOR & Inhibits $\mathrm{A} \beta$ deposition & [125] \\
\hline Arctigenin & $\mathrm{AKT} / \mathrm{mTOR}$ & Enhanced $\mathrm{A} \beta$ clearance & {$[130]$} \\
\hline \multicolumn{4}{|l|}{ Terpenes } \\
\hline Cubeben & $\mathrm{PI} 3 \mathrm{~K} / \mathrm{AMPK} / \mathrm{mTOR}$ & Decreased $\mathrm{A} \beta$ toxicity & {$[132]$} \\
\hline Geraniol & Increased Atg5-7-12 & Reduce $\alpha$-synuclein & {$[133]$} \\
\hline Cucurbitacin E & Regulate autophagy lysosomal pathway & Eliminate toxic deposits & {$[134]$} \\
\hline
\end{tabular}

A $\beta$ synthesis in HEK293 and N2a cells [123]. In mice, orally administered resveratrol crossed the blood-brain barrier, stimulated brain AMPK, and decreased $\mathrm{A} \beta$ deposition in the cerebral cortex [123]. The active component $\left(2,3,5,4^{\prime}\right.$ -tetrahydroxystilbene-2-O-glycoside) in Radix Polygoni Multiflori was reported to hinder autophagy by decreasing Beclin-1 levels, thus enhancing cognitive function [124], and carnosic acid stimulated autophagy by activating the AMPK/mTOR pathway and inhibited A $\beta$ deposition [125].

Resveratrol was observed to protect SH-SY5Y cells from rotenone-stimulated apoptosis and to increase $\alpha$-synuclein degradation in $\alpha$-synuclein-expressing PC12 cell lines by inducing autophagy. The mechanism of $\alpha$-synuclein degradation in a cellular model of PD involved the regulation of mammalian SIRT1 (silent information regulator 2)/AMPK (AMP-activated protein kinase), which diminished LC3-II protein levels and increased $\alpha$-synuclein clearance [126]. Resveratrol improved mitochondrial oxidative function by regulating the AMPK and SIRT1 pathways and increased macroautophagic flux by activating an LC3-independent pathway in early-onset PD fibroblasts [127]. In another study, resveratrol stimulated heme oxygenase-1 expression and inhibited dopaminergic cell death by controlling autophagic flux and, as a result, protected against rotenone-induced neuronal apoptosis in a PD model [128]. Corema album polyphenol fractions promoted nontoxic $\alpha$-synuclein formation and, thus, reduced its toxicity and aggregation in cells by enhancing autophagic flux and reducing oxidative stress [129]. In addition, Arctium lappa-derived arctigenin inhibited the generation and enhanced the clearance of $\mathrm{A} \beta$ by inducing autophagy by inhibiting AKT/mTOR signaling and AMPK/Raptor pathway activation in an animal model of $\mathrm{AD}$ [130].

5.6. Terpenes and Terpenoids. Recently, monoterpenes have been identified to be autophagy modulators [131]. Cubeben, a Piper cubeba sesquiterpene, decreased $\mathrm{A} \beta$ toxicity in primary neuronal cells, recovered autophagy via PI3K/AMPK signaling, and suppressed the inhibition of mTOR [132]. In 


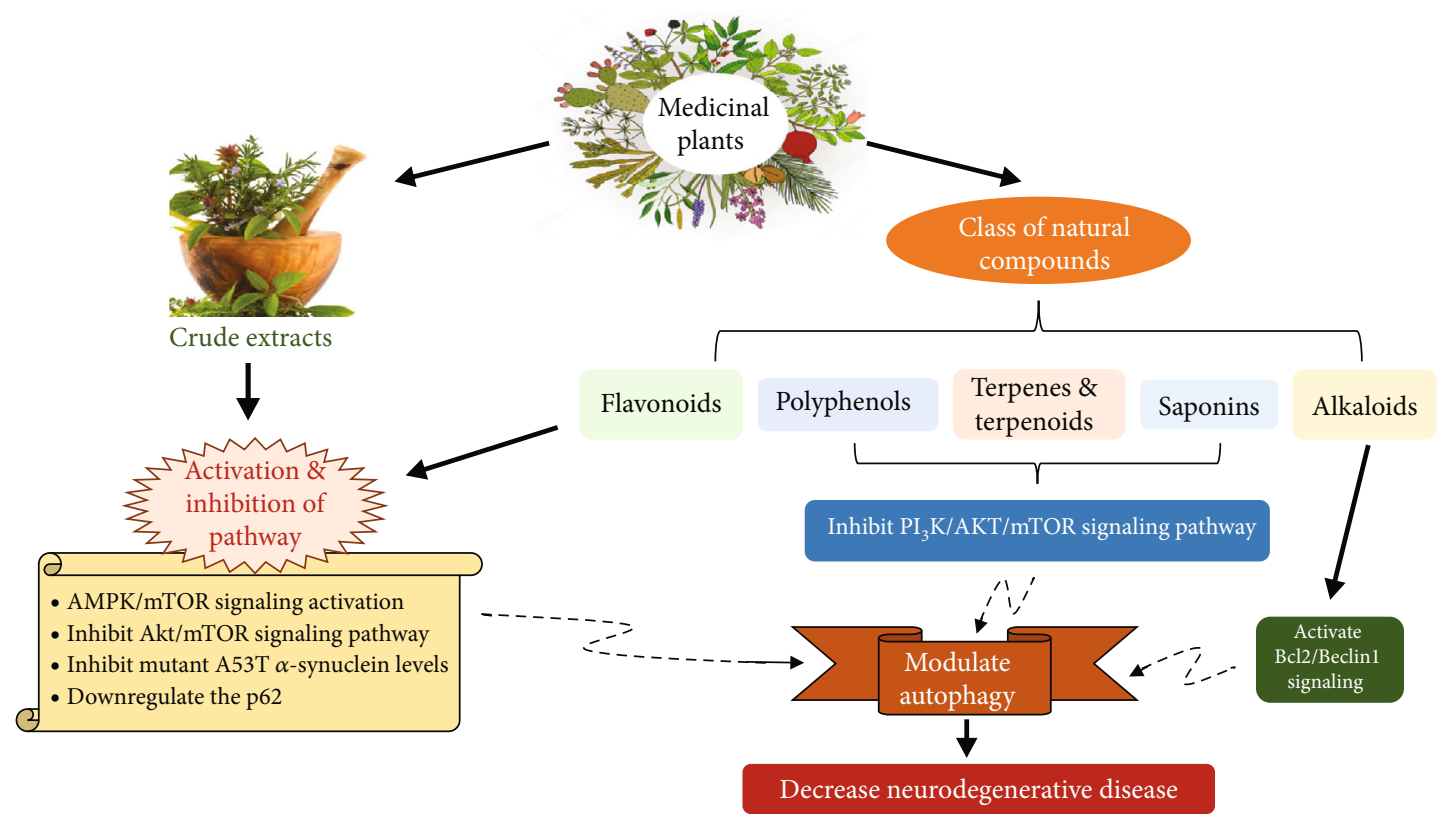

FIGURE 2: Different classes of natural compounds that modulate autophagy and suppress neurodegeneration by activating or inhibiting different molecular pathways.

a PD model, geraniol (an acyclic monoterpene) protected neurons against rotenone stress by restoring mitochondria, reducing $\alpha$-synuclein levels, and increasing autophagic flux [133]. Cucurbitacin E (a terpenoid phytosterol) partially protected PC12 neurons from PD simulating toxins, significantly decreased Beclin-1 autophagy, increased autophagosome activities, and eliminated toxic deposits [134].

\section{Nanomaterials, Autophagy, and NDs}

The need for innovative therapeutic approaches for NDs, as well as the limits imposed by the BBB, is driving the use of nanotechnology in the delivery of targeted drugs to the CNS. Because of their physical and chemical properties, nanomaterials can be excellent drug carriers to the brain $[135,136]$. Nanoparticles (NPs) stimulated intracellular autophagy, enhanced autophagosome breakdown, increased $\mathrm{A} \beta$ clearance in brain cell cultures, and decreased $\mathrm{A} \beta$-stimulated cytotoxicity [137]. The use of nanocarriers that encapsulate molecules may improve drug transport through the $\mathrm{BBB}$ in NDs and target key brain areas for regenerative processes [135]. Quercetin is a natural antioxidant that has a low capacity to cross the BBB and is easily eliminated. Recently, it has been demonstrated that quercetin-modified gold-palladium NPs increase the clearance of intracellular $\mathrm{A} \beta$ via autophagy activation and, thereby, decrease $\mathrm{A} \beta$ induced neurotoxicity [138]. This study paves the way for NPs to encapsulate natural products capable of modulating autophagy in the management of various NDs.

\section{Conclusion and Future Prospects}

Autophagy is an important process under normal and pathologic conditions. Studies have shown that the dysregulation of autophagy is involved in the pathogeneses of neurological disorders and suggested possible neuroprotective strategies to mitigate neurological disorders by managing the autophagy system. Several bioactive compounds derived from medicinal plants are believed to have the capabilities to control autophagy and treat NDs by targeting autophagic pathways. Regulation of the autophagic pathway is now viewed as an exciting drug developmental strategy because it is believed that the targeted control of autophagy offers a means of managing NDs.

\section{Conflicts of Interest}

The authors declare that they have no conflicts of interest.

\section{Authors' Contributions}

Inho Choi, Sibhghatulla Shaikh, and Khurshid Ahmad designed the study, and drafted the manuscript. Syed Sayeed Ahmad, Eun Ju Lee, Jeong Ho Lim, Mirza Masroor Ali Beg, and Amit K. Verma critically revised the manuscript. Sibhghatulla Shaikh and Khurshid Ahmad contributed equally to this work.

\section{Acknowledgments}

This research was supported by the Basic Science Research Program through the National Research Foundation of Korea (NRF) funded by the Ministry of Education (2020R1A6A 1A03044512) and by the NRF funded by the Korean government (MSIP: Grant No. NRF-2021R1A2C2004177).

\section{References}

[1] L. Yu, Y. Chen, and S. A. Tooze, "Autophagy pathway: cellular and molecular mechanisms," Autophagy, vol. 14, no. 2, pp. 207-215, 2018. 
[2] I. Dikic and Z. Elazar, "Mechanism and medical implications of mammalian autophagy," Nature Reviews. Molecular Cell Biology, vol. 19, no. 6, pp. 349-364, 2018.

[3] C. C. Tan, J. T. Yu, M. S. Tan, T. Jiang, X. C. Zhu, and L. Tan, "Autophagy in aging and neurodegenerative diseases: implications for pathogenesis and therapy," Neurobiology of Aging, vol. 35, no. 5, pp. 941-957, 2014.

[4] Z. Yin, C. Pascual, and D. J. Klionsky, "Autophagy: machinery and regulation," Microbial Cell, vol. 3, no. 12, pp. 588596, 2016.

[5] B. Levine and G. Kroemer, "Autophagy in the pathogenesis of disease," Cell, vol. 132, no. 1, pp. 27-42, 2008.

[6] E.-L. Eskelinen and P. Saftig, "Autophagy: a lysosomal degradation pathway with a central role in health and disease," Biochimica et Biophysica Acta (BBA)-Molecular Cell Research, vol. 1793, no. 4, pp. 664-673, 2009.

[7] Y. Wang, R. Singh, Y. Xiang, and M. J. Czaja, "Macroautophagy and chaperone-mediated autophagy are required for hepatocyte resistance to oxidant stress," Hepatology, vol. 52, no. 1, pp. 266-277, 2010.

[8] J. D. Mancias and A. C. Kimmelman, "Mechanisms of selective autophagy in normal physiology and cancer," Journal of Molecular Biology, vol. 428, no. 9, pp. 1659-1680, 2016.

[9] K. Inoue, J. Rispoli, H. Kaphzan et al., "Macroautophagy deficiency mediates age-dependent neurodegeneration through a phospho-tau pathway," Molecular Neurodegeneration, vol. 7, no. 1, pp. 1-13, 2012.

[10] S. Schuck, "Microautophagy - distinct molecular mechanisms handle cargoes of many sizes," Journal of Cell Science, vol. 133, no. 17, 2020.

[11] S. Kaushik and A. M. Cuervo, "The coming of age of chaperone-mediated autophagy," Nature Reviews Molecular Cell Biology, vol. 19, no. 6, pp. 365-381, 2018.

[12] I. E. Alfaro, A. Albornoz, A. Molina et al., "Chaperone mediated autophagy in the crosstalk of neurodegenerative diseases and metabolic disorders," Frontiers in Endocrinology, vol. 9, p. 778, 2019.

[13] R. A. Nixon, "The role of autophagy in neurodegenerative disease," Nature Medicine, vol. 19, no. 8, pp. 983-997, 2013.

[14] B. Boland, W. H. Yu, O. Corti et al., "Promoting the clearance of neurotoxic proteins in neurodegenerative disorders of ageing," Nature Reviews Drug Discovery, vol. 17, no. 9, pp. 660688, 2018.

[15] N. Mizushima, T. Yoshimori, and Y. Ohsumi, "The role of Atg proteins in autophagosome formation," Annual Review of Cell and Developmental Biology, vol. 27, no. 1, pp. 107$132,2011$.

[16] H. Nakatogawa, K. Suzuki, Y. Kamada, and Y. Ohsumi, "Dynamics and diversity in autophagy mechanisms: lessons from yeast," Nature Reviews. Molecular Cell Biology, vol. 10, no. 7, pp. 458-467, 2009.

[17] Y. Chen and D. J. Klionsky, "The regulation of autophagy unanswered questions," Journal of Cell Science, vol. 124, no. 2, pp. 161-170, 2011.

[18] R. J. Youle and D. P. Narendra, "Mechanisms of mitophagy," Nature Reviews. Molecular Cell Biology, vol. 12, no. 1, pp. 914, 2011.

[19] E. Itakura and N. Mizushima, "Characterization of autophagosome formation site by a hierarchical analysis of mammalian Atg proteins," Autophagy, vol. 6, no. 6, pp. 764-776, 2010 .
[20] D. Meng, A. R. Frank, and J. L. Jewell, "mTOR signaling in stem and progenitor cells," Development, vol. 145, no. 1, 2018.

[21] Y. Mugume, Z. Kazibwe, and D. C. Bassham, "Target of rapamycin in control of autophagy: puppet master and signal integrator," International Journal of Molecular Sciences, vol. 21, no. 21, p. 8259, 2020.

[22] S. Fan, B. Zhang, P. Luan et al., "PI3K/AKT/mTOR/p70S6K Pathway Is Involved in A $\beta 25-35$-Induced Autophagy," BioMed Research International, vol. 2015, Article ID 161020, 9 pages, 2015.

[23] Q. Li, Y. Liu, and M. Sun, "Autophagy and Alzheimer's disease," Cellular and Molecular Neurobiology, vol. 37, no. 3, pp. 377-388, 2017.

[24] R. Franco, E. Martinez-Pinilla, G. Navarro, and M. Zamarbide, "Potential of GPCRs to modulate MAPK and mTOR pathways in Alzheimer's disease," Progress in Neurobiology, vol. 149-150, pp. 21-38, 2017.

[25] F. Nazio and F. Cecconi, "mTOR, AMBRA1, and autophagy: an intricate relationship," Cell Cycle, vol. 12, no. 16, pp. 25242525, 2013.

[26] Z. Yang and D. J. Klionsky, "Mammalian autophagy: core molecular machinery and signaling regulation," Current Opinion in Cell Biology, vol. 22, no. 2, pp. 124-131, 2010.

[27] L. Ryskalin, F. Limanaqi, A. Frati, C. L. Busceti, and F. Fornai, "mTOR-related brain dysfunctions in neuropsychiatric disorders," International Journal of Molecular Sciences, vol. 19, no. 8, p. 2226, 2018.

[28] N. Mizushima, "The role of the Atg1/ULK1 complex in autophagy regulation," Current Opinion in Cell Biology, vol. 22, no. 2, pp. 132-139, 2010.

[29] C. H. Jung, C. B. Jun, S. H. Ro et al., "ULK-Atg13-FIP200 complexes mediate mTOR signaling to the autophagy machinery," Molecular Biology of the Cell, vol. 20, no. 7, pp. 1992-2003, 2009.

[30] S. Sarkar, R. A. Floto, Z. Berger et al., "Lithium induces autophagy by inhibiting inositol monophosphatase," The Journal of Cell Biology, vol. 170, no. 7, pp. 1101-1111, 2005.

[31] A. Williams, S. Sarkar, P. Cuddon et al., "Novel targets for Huntington's disease in an mTOR-independent autophagy pathway," Nature Chemical Biology, vol. 4, no. 5, pp. 295305, 2008 .

[32] S. F. Funderburk, B. K. Marcellino, and Z. Yue, "Cell "selfeating" (autophagy) mechanism in Alzheimer's disease," Mount Sinai Journal of Medicine: A Journal of Translational and Personalized Medicine, vol. 77, no. 1, pp. 5968, 2010.

[33] A. François, F. Terro, T. Janet, A. R. Bilan, M. Paccalin, and G. Page, "Involvement of interleukin-1 $\beta$ in the autophagic process of microglia: relevance to Alzheimer's disease," Journal of Neuroinflammation, vol. 10, no. 1, pp. 1-22, 2013.

[34] D. Glick, S. Barth, and K. F. Macleod, "Autophagy: cellular and molecular mechanisms," The Journal of Pathology, vol. 221, no. 1, pp. 3-12, 2010.

[35] D. C. Rubinsztein, G. Marino, and G. Kroemer, "Autophagy and aging," Cell, vol. 146, no. 5, pp. 682-695, 2011.

[36] A. J. Meijer, S. Lorin, E. F. Blommaart, and P. Codogno, "Regulation of autophagy by amino acids and MTOR-dependent signal transduction," Amino Acids, vol. 47, no. 10, pp. 2037-2063, 2015. 
[37] B. Boland, A. Kumar, S. Lee et al., "Autophagy induction and autophagosome clearance in neurons: relationship to autophagic pathology in Alzheimer's disease," The Journal of Neuroscience, vol. 28, no. 27, pp. 6926-6937, 2008.

[38] H. M. Shen and N. Mizushima, "At the end of the autophagic road: an emerging understanding of lysosomal functions in autophagy," Trends in Biochemical Sciences, vol. 39, no. 2, pp. 61-71, 2014.

[39] M. García-Arencibia, W. E. Hochfeld, P. P. Toh, and D. C. Rubinsztein, "Autophagy, a guardian against neurodegeneration," Seminars in Cell \& Developmental Biology, vol. 21, no. 7, pp. 691-698, 2010.

[40] M. Laplante and D. M. Sabatini, "mTOR signaling in growth control and disease," Cell, vol. 149, no. 2, pp. 274-293, 2012.

[41] R. Zoncu, A. Efeyan, and D. M. Sabatini, "mTOR: from growth signal integration to cancer, diabetes and ageing," Nature Reviews. Molecular Cell Biology, vol. 12, no. 1, pp. 21-35, 2011.

[42] D. J. Klionsky and S. D. Emr, "Autophagy as a regulated pathway of cellular degradation," Science, vol. 290, no. 5497, pp. 1717-1721, 2000.

[43] G. Kroemer, G. Marino, and B. Levine, "Autophagy and the integrated stress response," Molecular Cell, vol. 40, no. 2, pp. 280-293, 2010.

[44] B. Levine, N. Mizushima, and H. W. Virgin, "Autophagy in immunity and inflammation,” Nature, vol. 469, no. 7330, pp. 323-335, 2011.

[45] T. Saitoh and S. Akira, "Regulation of innate immune responses by autophagy-related proteins," The Journal of Cell Biology, vol. 189, no. 6, pp. 925-935, 2010.

[46] T. Saitoh, N. Fujita, T. Hayashi et al., "Atg9a controls dsDNA-driven dynamic translocation of STING and the innate immune response," Proceedings of the National Academy of Sciences of the United States of America, vol. 106, no. 49, pp. 20842-20846, 2009.

[47] H. Konno, K. Konno, and G. N. Barber, "Cyclic dinucleotides trigger ULK1 (ATG1) phosphorylation of STING to prevent sustained innate immune signaling," Cell, vol. 155, no. 3, pp. 688-698, 2013.

[48] Q. Liang, G. J. Seo, Y. J. Choi et al., "Crosstalk between the cGAS DNA sensor and beclin-1 autophagy protein shapes innate antimicrobial immune responses," Cell Host \& Microbe, vol. 15, no. 2, pp. 228-238, 2014.

[49] M. C. Tal, M. Sasai, H. K. Lee, B. Yordy, G. S. Shadel, and A. Iwasaki, "Absence of autophagy results in reactive oxygen species-dependent amplification of RLR signaling," Proceedings of the National Academy of Sciences of the United States of America, vol. 106, no. 8, pp. 2770-2775, 2009.

[50] S. Paul, A. K. Kashyap, W. Jia, Y. W. He, and B. C. Schaefer, "Selective autophagy of the adaptor protein Bcl10 modulates T cell receptor activation of NF- $\kappa$ B," Immunity, vol. 36, no. 6 , pp. 947-958, 2012.

[51] S. Trunova and E. Giniger, "Absence of the Cdk5 activator p35 causes adult-onset neurodegeneration in the central brain of Drosophila," Disease Models \& Mechanisms, vol. 5, no. 2, pp. 210-219, 2012.

[52] J. Spurrier, A. K. Shukla, K. McLinden, K. Johnson, and E. Giniger, "Altered expression of the Cdk5 activator-like protein, Cdk5alpha, causes neurodegeneration, in part by accelerating the rate of aging," Disease Models \& Mechanisms, vol. 11, no. 3, 2018.
[53] E. Czirr and T. Wyss-Coray, "The immunology of neurodegeneration," The Journal of Clinical Investigation, vol. 122, no. 4, pp. 1156-1163, 2012.

[54] A. Plaza-Zabala, V. Sierra-Torre, and A. Sierra, "Autophagy and microglia: novel partners in neurodegeneration and aging," International Journal of Molecular Sciences, vol. 18, no. 3, p. 598, 2017.

[55] M. H. Cho, K. Cho, H. J. Kang et al., "Autophagy in microglia degrades extracellular $\beta$-amyloid fibrils and regulates the NLRP3 inflammasome," Autophagy, vol. 10, no. 10, pp. 1761-1775, 2014.

[56] M. Madill, K. McDonagh, J. Ma et al., “Amyotrophic lateral sclerosis patient iPSC-derived astrocytes impair autophagy via non-cell autonomous mechanisms," Molecular Brain, vol. 10, no. 1, p. 22, 2017.

[57] H. Phatnani and T. Maniatis, "Astrocytes in neurodegenerative disease," Cold Spring Harbor Perspectives in Biology, vol. 7, no. 6, 2015.

[58] M. Qaisiya, P. Mardesic, B. Pastore, C. Tiribelli, and C. Bellarosa, "The activation of autophagy protects neurons and astrocytes against bilirubin-induced cytotoxicity," Neuroscience Letters, vol. 661, pp. 96-103, 2017.

[59] S. Wang, B. Li, H. Qiao et al., "Autophagy-related gene Atg5 is essential for astrocyte differentiation in the developing mouse cortex," EMBO Reports, vol. 15, no. 10, pp. 10531061, 2014.

[60] B. Caballero, Y. Wang, A. Diaz et al., "Interplay of pathogenic forms of human tau with different autophagic pathways," Aging Cell, vol. 17, no. 1, p. e12692, 2018.

[61] L. Vaillant-Beuchot, A. Mary, R. Pardossi-Piquard et al., "Accumulation of amyloid precursor protein C-terminal fragments triggers mitochondrial structure, function, and mitophagy defects in Alzheimer's disease models and human brains," Acta Neuropathologica, vol. 141, no. 1, pp. 39-65, 2021.

[62] A. E. González, V. C. Muñoz, V. A. Cavieres et al., “Autophagosomes cooperate in the degradation of intracellular Cterminal fragments of the amyloid precursor proteinviathe MVB/lysosomal pathway," The FASEB Journal, vol. 31, no. 6, pp. 2446-2459, 2017.

[63] I. Lauritzen, R. Pardossi-Piquard, A. Bourgeois et al., "Intraneuronal aggregation of the $\beta$-CTF fragment of APP (C99) induces A $\beta$-independent lysosomal-autophagic pathology," Acta Neuropathologica, vol. 132, no. 2, pp. 257-276, 2016.

[64] L. K. Hein, P. M. Apaja, K. Hattersley et al., "A novel fluorescent probe reveals starvation controls the commitment of amyloid precursor protein to the lysosome," Biochimica et Biophysica Acta (BBA) - Molecular Cell Research, vol. 1864, no. 10, pp. 1554-1565, 2017.

[65] A. Halle, V. Hornung, G. C. Petzold et al., "The NALP3 inflammasome is involved in the innate immune response to amyloid- $\beta$," Nature Immunology, vol. 9, no. 8, pp. 857$865,2008$.

[66] D. S. Yang, P. Stavrides, M. Saito et al., "Defective macroautophagic turnover of brain lipids in the TgCRND8 Alzheimer mouse model: prevention by correcting lysosomal proteolytic deficits," Brain, vol. 137, no. 12, pp. 3300-3318, 2014.

[67] E. Kesidou, R. Lagoudaki, O. Touloumi, K. N. Poulatsidou, and C. Simeonidou, "Autophagy and neurodegenerative disorders," Neural Regeneration Research, vol. 8, no. 24, pp. 2275-2283, 2013. 
[68] Y. S. Rajawat and I. Bossis, "Autophagy in aging and in neurodegenerative disorders," Hormones (Athens, Greece), vol. 7, no. 1, pp. 46-61, 2008.

[69] T. Hamano, T. F. Gendron, E. Causevic et al., "Autophagiclysosomal perturbation enhances tau aggregation in transfectants with induced wild-type tau expression," The European Journal of Neuroscience, vol. 27, no. 5, pp. 1119-1130, 2008.

[70] J. W. Steele and S. Gandy, "Latrepirdine (Dimebon ${ }^{\circledR}$ ), a potential Alzheimer therapeutic, regulates autophagy and neuropathology in an Alzheimer mouse model," Autophagy, vol. 9, no. 4, pp. 617-618, 2013.

[71] A. Richardson, V. Galvan, A. L. Lin, and S. Oddo, "How longevity research can lead to therapies for Alzheimer's disease: the rapamycin story," Experimental Gerontology, vol. 68, pp. 51-58, 2015.

[72] L. Zhang, L. Wang, R. Wang et al., "Evaluating the effectiveness of GTM-1, rapamycin, and carbamazepine on autophagy and Alzheimer disease," Medical Science Monitor, vol. 23, pp. 801-808, 2017.

[73] A. Caccamo, E. Ferreira, C. Branca, and S. Oddo, "Retracted article: p62 improves AD-like pathology by increasing autophagy," Molecular Psychiatry, vol. 22, no. 6, pp. 865873, 2017.

[74] R. Abdullah, I. Basak, K. S. Patil, G. Alves, J. P. Larsen, and S. G. Moller, "Parkinson's disease and age: the obvious but largely unexplored link," Experimental Gerontology, vol. 68, pp. 33-38, 2015.

[75] Y. Cai, J. Arikkath, L. Yang, M. L. Guo, P. Periyasamy, and S. Buch, "Interplay of endoplasmic reticulum stress and autophagy in neurodegenerative disorders," Autophagy, vol. 12, no. 2, pp. 225-244, 2016.

[76] A. M. Cuervo, L. Stefanis, R. Fredenburg, P. T. Lansbury, and D. Sulzer, "Impaired degradation of mutant alpha-synuclein by chaperone-mediated autophagy," Science, vol. 305, no. 5688, pp. 1292-1295, 2004.

[77] B. Dehay, J. Bove, N. Rodriguez-Muela et al., "Pathogenic lysosomal depletion in Parkinson's disease," The Journal of Neuroscience, vol. 30, no. 37, pp. 12535-12544, 2010.

[78] M. L. Hebron, I. Lonskaya, and C. E. Moussa, "Nilotinib reverses loss of dopamine neurons and improves motor behavior via autophagic degradation of $\alpha$-synuclein in Parkinson's disease models," Human Molecular Genetics, vol. 22, no. 16, pp. 3315-3328, 2013.

[79] J. L. Webb, B. Ravikumar, J. Atkins, J. N. Skepper, and D. C. Rubinsztein, " $\alpha$-Synuclein is degraded by both autophagy and the proteasome*," The Journal of Biological Chemistry, vol. 278, no. 27, pp. 25009-25013, 2003.

[80] A. R. Winslow, C. W. Chen, S. Corrochano et al., " $\alpha$-Synuclein impairs macroautophagy: implications for Parkinson's disease," The Journal of Cell Biology, vol. 190, no. 6, pp. 1023-1037, 2010.

[81] M. Martinez-Vicente, Z. Talloczy, S. Kaushik et al., "Dopamine-modified alpha-synuclein blocks chaperone-mediated autophagy," The Journal of Clinical Investigation, vol. 118, no. 2, pp. 777-788, 2008.

[82] P. Pasinelli and R. H. Brown, "Molecular biology of amyotrophic lateral sclerosis: insights from genetics," Nature Reviews. Neuroscience, vol. 7, no. 9, pp. 710-723, 2006.

[83] N. Morimoto, M. Nagai, Y. Ohta et al., "Increased autophagy in transgenic mice with a G93A mutant SOD1 gene," Brain Research, vol. 1167, pp. 112-117, 2007.
[84] M. Filimonenko, S. Stuffers, C. Raiborg et al., "Functional multivesicular bodies are required for autophagic clearance of protein aggregates associated with neurodegenerative disease," The Journal of Cell Biology, vol. 179, no. 3, pp. 485500, 2007.

[85] H. X. Deng, W. Chen, S. T. Hong et al., "Mutations in UBQLN2 cause dominant X-linked juvenile and adult-onset ALS and ALS/dementia," Nature, vol. 477, no. 7363, pp. 211-215, 2011.

[86] E. J. Lee, S. Shaikh, K. Ahmad et al., "Isolation and characterization of compounds from Glycyrrhiza uralensis as therapeutic agents for the muscle disorders," International Journal of Molecular Sciences, vol. 22, no. 2, 2021.

[87] M. H. Baig, A. T. Jan, G. Rabbani et al., "Methylglyoxal and advanced glycation end products: insight of the regulatory machinery affecting the myogenic program and of its modulation by natural compounds," Scientific Reports, vol. 7, no. 1, p. 5916, 2017.

[88] M. J. R. Howes, C. L. Quave, J. Collemare et al., "Molecules from nature: reconciling biodiversity conservation and global healthcare imperatives for sustainable use of medicinal plants and fungi," Plants, People, Planet, vol. 2, no. 5, pp. 463-481, 2020.

[89] S. Shaikh, E. J. Lee, K. Ahmad, S. S. Ahmad, J. H. Lim, and I. Choi, "A comprehensive review and perspective on natural sources as dipeptidyl peptidase-4 inhibitors for management of diabetes," Pharmaceuticals (Basel), vol. 14, no. 6, p. 591, 2021.

[90] S. G. Sreenivasmurthy, J. Y. Liu, J. X. Song et al., "Neurogenic traditional Chinese medicine as a promising strategy for the treatment of Alzheimer's disease," International Journal of Molecular Sciences, vol. 18, no. 2, p. 272, 2017.

[91] S. F. Wang, M. Y. Wu, C. Z. Cai, M. Li, and J. H. Lu, "Autophagy modulators from traditional Chinese medicine: mechanisms and therapeutic potentials for cancer and neurodegenerative diseases," Journal of Ethnopharmacology, vol. 194, pp. 861-876, 2016.

[92] S. S. Ahmad, M. B. Khan, K. Ahmad et al., "Biocomputational screening of natural compounds against acetylcholinesterase," Molecules, vol. 26, no. 9, p. 2641, 2021.

[93] A. G. Wu, V. K. Wong, S. W. Xu et al., "Onjisaponin B derived from Radix Polygalae enhances autophagy and accelerates the degradation of mutant $\alpha$-synuclein and Huntingtin in PC-12 cells," International Journal of Molecular Sciences, vol. 14, no. 11, pp. 22618-22641, 2013.

[94] H. Zhao, Z. C. Wang, K. F. Wang, and X. Y. Chen, “A $\beta$ peptide secretion is reduced by Radix Polygalae-induced autophagy via activation of the AMPK/mTOR pathway," Molecular Medicine Reports, vol. 12, no. 2, pp. 2771-2776, 2015.

[95] K. Dutta, P. Patel, and J. P. Julien, "Protective effects of Withania somnifera extract in SOD1 ${ }^{\text {G93A }}$ mouse model of amyotrophic lateral sclerosis," Experimental Neurology, vol. 309, pp. 193-204, 2018.

[96] X. Liu, W. Hao, Y. Qin et al., "Long-term treatment with Ginkgo biloba extract EGb 761 improves symptoms and pathology in a transgenic mouse model of Alzheimer 's disease," Brain, Behavior, and Immunity, vol. 46, pp. 121-131, 2015.

[97] Y. Fan, N. Wang, A. Rocchi et al., "Identification of natural products with neuronal and metabolic benefits through autophagy induction," Autophagy, vol. 13, no. 1, pp. 41-56, 2017. 
[98] X. Guo, J. Lv, J. Lu et al., "Protopanaxadiol derivative DDPU improves behavior and cognitive deficit in $\mathrm{AD}$ mice involving regulation of both ER stress and autophagy," Neuropharmacology, vol. 130, pp. 77-91, 2018.

[99] D. Kaufmann, A. Kaur Dogra, A. Tahrani, F. Herrmann, and M. Wink, "Extracts from traditional Chinese medicinal plants inhibit acetylcholinesterase, a known Alzheimer's disease target," Molecules, vol. 21, no. 9, p. 1161, 2016.

[100] K. Umezawa, I. Kojima, S. Simizu et al., "Therapeutic activity of plant-derived alkaloid conophylline on metabolic syndrome and neurodegenerative disease models," Human Cell, vol. 31, no. 2, pp. 95-101, 2018.

[101] L. S. Li, Y. L. Lu, J. Nie et al., "Dendrobium nobile Lindl alkaloid, a novel autophagy inducer, protects against axonal degeneration induced by $\mathrm{A} \beta 25-35$ in hippocampus neurons in vitro," CNS Neuroscience \& Therapeutics, vol. 23, no. 4, pp. 329-340, 2017.

[102] M. Huang, X. Jiang, Y. Liang, Q. Liu, S. Chen, and Y. Guo, "Berberine improves cognitive impairment by promoting autophagic clearance and inhibiting production of $\beta$-amyloid in APP/tau/PS1 mouse model of Alzheimer's disease," Experimental Gerontology, vol. 91, pp. 25-33, 2017.

[103] X. Wang, R. Wang, D. Xing et al., "Kinetic difference of berberine between hippocampus and plasma in rat after intravenous administration of Coptidis rhizoma extract," Life Sciences, vol. 77, no. 24, pp. 3058-3067, 2005.

[104] M. Neumann, D. M. Sampathu, L. K. Kwong et al., "Ubiquitinated TDP-43 in frontotemporal lobar degeneration and amyotrophic lateral sclerosis," Science, vol. 314, no. 5796, pp. 130-133, 2006.

[105] T. Arai, M. Hasegawa, H. Akiyama et al., "TDP-43 is a component of ubiquitin-positive tau-negative inclusions in frontotemporal lobar degeneration and amyotrophic lateral sclerosis," Biochemical and Biophysical Research Communications, vol. 351, no. 3, pp. 602-611, 2006.

[106] C. F. Chang, Y. C. Lee, K. H. Lee et al., "Therapeutic effect of berberine on TDP-43-related pathogenesis in FTLD and ALS," Journal of Biomedical Science, vol. 23, no. 1, p. 72, 2016.

[107] L. L. Chen, J. X. Song, J. H. Lu et al., "Corynoxine, a natural autophagy enhancer, promotes the clearance of alphasynuclein via Akt/mTOR pathway," Journal of Neuroimmune Pharmacology, vol. 9, no. 3, pp. 380-387, 2014.

[108] J. H. Lu, J. Q. Tan, S. S. Durairajan et al., "Isorhynchophylline, a natural alkaloid, promotes the degradation of alphasynuclein in neuronal cells via inducing autophagy," Autophagy, vol. 8, no. 1, pp. 98-108, 2012.

[109] K. W. Hsueh, T. W. Chiou, S. F. Chiang et al., "Autophagic down-regulation in motor neurons remarkably prolongs the survival of ALS mice," Neuropharmacology, vol. 108, pp. 152-160, 2016.

[110] Y. Sasazawa, N. Sato, K. Umezawa, and S. Simizu, "Conophylline protects cells in cellular models of neurodegenerative diseases by inducing mammalian target of rapamycin (mTOR)-independent autophagy*," The Journal of Biological Chemistry, vol. 290, no. 10, pp. 6168-6178, 2015.

[111] H. S. Song, S. Jang, and S. C. Kang, "Bavachalcone from Cullen corylifolium induces apoptosis and autophagy in HepG2 cells," Phytomedicine, vol. 40, pp. 37-47, 2018.

[112] X. M. Zhang, D. Q. An, L. L. Guo, N. H. Yang, and H. Zhang, "Identification and screening of active components from Ziziphora clinopodioides Lam. in regulating autophagy,"
Natural Product Research, vol. 33, no. 17, pp. 2549-2553, 2019.

[113] X. Song, B. Liu, L. Cui et al., "Silibinin ameliorates anxiety/depression-like behaviors in amyloid $\beta$-treated rats by upregulating $\mathrm{BDNF} / \mathrm{TrkB}$ pathway and attenuating autophagy in hippocampus," Physiology \& Behavior, vol. 179, pp. 487493, 2017.

[114] Y. Zhu and J. Wang, "Wogonin increases $\beta$-amyloid clearance and inhibits tau phosphorylation via inhibition of mammalian target of rapamycin: potential drug to treat Alzheimer's disease," Neurological Sciences, vol. 36, no. 7, pp. 1181-1188, 2015.

[115] S. M. Huang, S. Y. Tsai, J. A. Lin, C. H. Wu, and G. C. Yen, "Cytoprotective effects of hesperetin and hesperidin against amyloid $\beta$-induced impairment of glucose transport through downregulation of neuronal autophagy," Molecular Nutrition \& Food Research, vol. 56, no. 4, pp. 601-609, 2012.

[116] G. Filomeni, I. Graziani, D. De Zio et al., "Neuroprotection of kaempferol by autophagy in models of rotenone-mediated acute toxicity: possible implications for Parkinson's disease," Neurobiology of Aging, vol. 33, no. 4, pp. 767-785, 2012.

[117] C. Wang, X. Zhang, Z. Teng, T. Zhang, and Y. Li, "Downregulation of PI3K/Akt/mTOR signaling pathway in curcumininduced autophagy in APP/PS1 double transgenic mice," European Journal of Pharmacology, vol. 740, pp. 312-320, 2014.

[118] L. Zhang, Y. Fang, X. Cheng et al., "The Potential Protective Effect of Curcumin on Amyloid- $\beta$-42 Induced Cytotoxicity in HT-22 Cells," BioMed Research International, vol. 2018, Article ID 8134902, 8 pages, 2018.

[119] T. Jaroonwitchawan, N. Chaicharoenaudomrung, J. Namkaew, and P. Noisa, "Curcumin attenuates paraquatinduced cell death in human neuroblastoma cells through modulating oxidative stress and autophagy," Neuroscience Letters, vol. 636, pp. 40-47, 2017.

[120] K. Drygalski, E. Fereniec, K. Korycinski et al., "Resveratrol and Alzheimer's disease. From molecular pathophysiology to clinical trials," Experimental Gerontology, vol. 113, pp. 36-47, 2018.

[121] T. F. Jiang, Y. J. Zhang, H. Y. Zhou et al., "Curcumin ameliorates the neurodegenerative pathology in A53T $\alpha$-synuclein cell model of Parkinson's disease through the downregulation of $\mathrm{mTOR} / \mathrm{p} 70 \mathrm{~S} 6 \mathrm{~K}$ signaling and the recovery of macroautophagy," Journal of Neuroimmune Pharmacology, vol. 8, no. 1, pp. 356-369, 2013.

[122] H. Wang, T. Jiang, W. Li, N. Gao, and T. Zhang, "Resveratrol attenuates oxidative damage through activating mitophagy in an in vitro model of Alzheimer 's disease," Toxicology Letters, vol. 282, pp. 100-108, 2018.

[123] V. Vingtdeux, L. Giliberto, H. Zhao et al., "AMP-activated protein kinase signaling activation by resveratrol modulates amyloid- $\beta$ peptide metabolism," The Journal of Biological Chemistry, vol. 285, no. 12, pp. 9100-9113, 2010.

[124] H. Luo, Y. Li, J. Guo et al., "Tetrahydroxy stilbene glucoside improved the behavioral disorders of APP695V717I transgenic mice by inhibiting the expression of Beclin-1 and LC3-II," Journal of Traditional Chinese Medicine, vol. 35, no. 3, pp. 295-300, 2015.

[125] J. Liu, H. Su, and Q. M. Qu, "Carnosic acid prevents betaamyloid-induced injury in human neuroblastoma SH-SY5Y cells via the induction of autophagy," Neurochemical Research, vol. 41, no. 9, pp. 2311-2323, 2016. 
[126] Y. Wu, X. Li, J. X. Zhu et al., "Resveratrol-activated AMPK/SIRT1/autophagy in cellular models of Parkinson's disease," Neurosignals, vol. 19, no. 3, pp. 163-174, 2011.

[127] A. Ferretta, A. Gaballo, P. Tanzarella et al., "Effect of resveratrol on mitochondrial function: implications in parkinassociated familiar Parkinson's disease," Biochimica et Biophysica Acta, vol. 1842, no. 7, pp. 902-915, 2014.

[128] T. K. Lin, S. D. Chen, Y. C. Chuang et al., "Resveratrol partially prevents rotenone-induced neurotoxicity in dopaminergic SH-SY5Y cells through induction of heme oxygenase-1 dependent autophagy," International Journal of Molecular Sciences, vol. 15, no. 1, pp. 1625-1646, 2014.

[129] D. Macedo, L. Tavares, G. J. McDougall et al., “(Poly)phenols protect from $\alpha$-synuclein toxicity by reducing oxidative stress and promoting autophagy," Human Molecular Genetics, vol. 24, no. 6, pp. 1717-1732, 2015.

[130] Z. Zhu, J. Yan, W. Jiang et al., “Arctigenin effectively ameliorates memory impairment in Alzheimer's disease model mice targeting both $\beta$-amyloid production and clearance," The Journal of Neuroscience, vol. 33, no. 32, pp. 13138-13149, 2013.

[131] M. Ashrafizadeh, Z. Ahmadi, T. Farkhondeh, and S. Samarghandian, "Autophagy as a molecular target of quercetin underlying its protective effects in human diseases," Archives of Physiology and Biochemistry, pp. 1-9, 2019.

[132] X. Li, J. Song, and R. Dong, "Cubeben induces autophagy via PI3K-AKT-mTOR pathway to protect primary neurons against amyloid beta in Alzheimer's disease," Cytotechnology, vol. 71, no. 3, pp. 679-686, 2019.

[133] K. R. Rekha and R. Inmozhi Sivakamasundari, "Geraniol protects against the protein and oxidative stress induced by rotenone in an in vitro model of Parkinson's disease," Neurochemical Research, vol. 43, no. 10, pp. 1947-1962, 2018.

[134] A. M. Arel-Dubeau, F. Longpre, J. Bournival et al., "Cucurbitacin E has neuroprotective properties and autophagic modulating activities on dopaminergic neurons," Oxidative Medicine and Cellular Longevity, vol. 2014, Article ID 425496, 15 pages, 2014.

[135] X. Niu, J. Chen, and J. Gao, "Nanocarriers as a powerful vehicle to overcome blood-brain barrier in treating neurodegenerative diseases: focus on recent advances," Asian Journal of Pharmaceutical Sciences, vol. 14, no. 5, pp. 480-496, 2019.

[136] G. Modi, V. Pillay, and Y. E. Choonara, "Advances in the treatment of neurodegenerative disorders employing nanotechnology," Annals of the New York Academy of Sciences, vol. 1184, no. 1, pp. 154-172, 2010.

[137] F. Gao, J. Zhao, P. Liu et al., "Preparation and in vitro evaluation of multi-target-directed selenium-chondroitin sulfate nanoparticles in protecting against the Alzheimer's disease," International Journal of Biological Macromolecules, vol. 142, pp. 265-276, 2020.

[138] Y. Liu, H. Zhou, T. Yin et al., "Quercetin-modified goldpalladium nanoparticles as a potential autophagy inducer for the treatment of Alzheimer's disease," Journal of Colloid and Interface Science, vol. 552, pp. 388-400, 2019. 